\title{
Rol de trabajo social en atención a adultos mayores que reciben diálisis en un hospital público de Ecuador, enero 2019
}

\author{
Dra. Soraya del Pilar Carranco Madrid Ph.D \\ spilarcm@hotmail.com \\ https://orcid.org/0000-0001-9039-5066 \\ Universidad Central del Ecuador / Quito - Ecuador \\ Lcda. Mónica Gualpa Sisalema \\ monika10961@gmail.com \\ Universidad Central del Ecuador / Quito - Ecuador \\ MSc. Jorge Antonio Piedra Rosales \\ japiedra@uce.edu.ec \\ https://orcid.org/0000-0001-9308-5215 \\ Universidad Central del Ecuador / Quito - Ecuador
}

\section{RESUMEN}

El presente estudio investigativo evidencia en términos generales, el Rol trascendental que cumple la Trabajadora Social en el área de salud, frente a la realidad que viven los adultos mayores que padecen Insuficiencia Renal Crónica Terminal, que les obliga a recibir tratamiento sustitutivo de la función renal (hemodiálisis) de por vida, a menos que exista la posibilidad de un trasplante renal. Se dejan expuestas varias metodologías, métodos, técnicas e instrumentos de intervención aplicados en la atención directa a los pacientes insuficientes renales. Adicionalmente, se abordan aspectos relacionados a datos sociodemográficos y datos de salud, tiempo en diálisis, conformación familiar, actividad física, atención externa y atención complementaria. Se procura contribuir con un abordaje real del Rol que cumple el Trabajador Social en el proceso de atención en salud, particularmente en atención a pacientes nefrópatas adultos mayores y su entorno familiar.

Palabras Clave: trabajo social; adultos mayores; insuficiencia renal; diálisis, familia. 


\title{
Role of Social Work in care for older adults receiving dialysis in a public hospital in Ecuador, january 2019
}

\begin{abstract}
The present investigative study shows in general terms, the transcendental role played by the Social Worker in the health area, compared to the reality experienced by older adults who suffer from Terminal Chronic Renal Failure, which forces them to receive replacement treatment for kidney function (hemodialysis) for life, unless there is a possibility of a kidney transplant. Various methodologies, methods, techniques and instruments of intervention applied in the direct care of kidney failure patients are exposed. Additionally, aspects related too sociodemographic data an health data, time on dialysis, family conformation physical activity external care and complementary care are addressed. It seeks to contribute with a real approach to the Rol of the Social Worker in the health care process, particularly in care fore elderly nephropathic patients and their family environment.
\end{abstract}

Keywords: social work; older adults; renal insufficiency; dialysis; family.

Artículo recibido: 05 octubre. 2021 Aceptado para publicación: 02 noviembre 2021

Correspondencia: spilarcm@ hotmail.com Conflictos de Interés: Ninguna que declarar 


\section{INTRODUCCIÓN}

El Trabajo Social es una disciplina y profesión de las Ciencias Sociales basada en la práctica, que tiene como objetivo promover el desarrollo integral del individuo, orientar a la resolución de problemas sociales mediante el uso de metodologías, modelos, técnicas e instrumentos propios de Trabajo Social, partiendo desde los principios de derechos al momento de la intervención profesional en los diferentes campos de acción como: salud, educación, protección, laboral y comunidad, que se realiza de manera directa con las personas y su entorno familiar, social, institucional y territorial, como expresa la Federación Internacional de Trabajadores Sociales (2020):

El trabajo social es una profesión basada en la práctica y una disciplina académica que promueve el cambio y el desarrollo social, la cohesión social, y el fortalecimiento y la liberación de las personas. Los principios de la justicia social, los derechos humanos, la responsabilidad colectiva y el respeto a la diversidad son fundamentales para el trabajo social. Respaldada por las teorías del trabajo social, las ciencias sociales, las humanidades y los conocimientos indígenas, el trabajo social involucra a las personas y las estructuras para hacer frente a desafíos de la vida y aumentar el bienestar.

El Trabajador Social en el área de Salud, forma parte del equipo multidisciplinario y aporta con el diagnóstico social, que se realiza a través de la interacción directa con los individuos que poseen alguna enfermedad, misma que permite reconocer los componentes de riesgo social como: entorno familiar, social e institucional, debido a que son grupos que influyen para la recuperación del paciente, tal como describe Ávila (2020):

Es una pieza clave del equipo multidisciplinar. Existen especializaciones en el ámbito clínico o psiquiátrico en trabajo social. Se pueden utilizar los diversos marcos teóricos, metodológicos, instrumentales y tecnológicos acorde a las necesidades institucionales. Hay puestos operativos, de coordinación y dirección en trabajo social hospitalario. (pág. 5)

El Trabajador Social en el Área Médica, desarrolla su intervención directa con los pacientes y sus familiares, con el propósito de orientar a la resolución de problemas, 
contribuir y sensibilizar sobre la realidad social que atraviesan dentro de la sociedad, a través de la implementación de programas y proyectos preventivos. Además, el profesional utiliza diferentes metodologías, técnicas e instrumentos que permiten analizar la realidad social, identificar y detectar las necesidades y los problemas socio-familiares a los que están expuestos los pacientes.

En el ámbito hospitalario el Rol de Trabajo Social con las personas de la tercera edad, es muy amplio por las actividades que se ejecutan mediante la intervención directa con los individuos y los determinantes sociales que influyen en la salud de los pacientes como: su ámbito familiar, social e institucional en la cual se desarrolla, con la finalidad de promover el bienestar integral de los usuarios y la participación en los diferentes programas de prevención fundamentada en las necesidades sociales y las situaciones que atraviesan los adultos mayores dentro de la sociedad.

El término adulto mayor hace referencia a la población entre los 60 y 74 años de edad en adelante, evita la utilización de términos ofensivos y groseros al momento de referirse o dirigirse a los mismos, y es considerada como la última etapa de vida en la que el cuerpo y las funciones cognitivas comienzan a deteriorarse y afectan de una manera directa la calidad de vida y salud del individuo, así como plantea la Organización Mundial de la Salud (s.f):

Se entiende el término adulto mayor a las personas que se encuentran entre los 60 y 74 años de edad. Se ha utilizado el término de "Adulto mayor", para hacer referencia a la etapa de la vejez humana y evitar el uso de términos peyorativos y humillantes, de tal manera que se entienda que a partir de los 65 años se es con dignidad un adulto mayor. Los adultos mayores pertenecen al grupo de atención prioritaria por su mayor grado de vulnerabilidad y son seres humanos con dignidad y sujetos de derechos que deben ser incluidos en los beneficios, programas sociales y en la creación de condiciones de igualdad y equidad en las instituciones públicas y privadas.

Actualmente en la sociedad, un sector de la población, que considera desde su visión a los adultos mayores, como personas improductivas, debido a que en la mayoría de los casos son limitadas a vivir un sedentarismo que implica realizar el menor número de actividades posibles, generando así personas de la tercera edad dependientes de sus familias, lo que constituyen una carga familiar y más cuando presenta alguna enfermedad. 
De la misma forma, la insuficiencia renal, que es una enfermedad que ocurre cuando los riñones no tienen un normal funcionamiento y más cuando los que poseen son los adultos mayores, se puede asociar a otras enfermedades vasculares por las complicaciones que se presentan, ya que los riñones son uno de los órganos muy importantes en el organismo por el funcionamiento que consiste en; filtrar y eliminar los abundantes líquidos del cuerpo, mantener el contenido corporal, secretar hormonas y modificar sustancias. También esta enfermedad puede provocar cambios que afecten a nivel individual, familiar, social y la calidad de vida del paciente, como señala la Sociedad Radiológica de Norteamérica (s.f):

Describe una situación en la que los riñones han perdido la capacidad de llevar a cabo estas funciones eficazmente. La acumulación en los de niveles de residuos puede causar un desequilibrio químico en la sangre, que puede ser fatal si no se trata. Los pacientes con insuficiencia renal pueden desarrollar, con el tiempo, un recuento sanguíneo bajo o huesos débiles.

Hay que destacar que, la enfermedad renal produce cambios en el estado emocional, social y familiar del usuario, ya que el cuerpo no tiene un equilibrio adecuado debido a la enfermedad, donde el individuo debe enfrentar a un nuevo estilo de vida y cambiar sus hábitos alimenticios y su rutita diaria, debido al tratamiento que debe recibir en la Institución de Salud.

Es decir, los pacientes deben recibir el tratamiento bi o trisemanal de hemodiálisis en las Instituciones de Salud en el área de diálisis, misma que consiste en eliminar y purificar el exceso de toxinas que se encuentran en el organismo de los pacientes, debido a la retención de los líquidos por la dificultad que se presenta debido al mal funcionamiento de los riñones. Dicho proceso se realiza periódicamente a través de un equipo específico y tecnológico, que tiene una duración de 4 a 5 horas, donde los usuarios deben permanecer acostados o sentados hasta que se termine el tratamiento.

La participación del Trabajador Social con los adultos mayores que reciben diálisis es fundamental, puesto que pertenecen al grupo de atención prioritaria por su mayor grado de vulnerabilidad y se debe tener en cuenta que son sujetos de derechos en el proceso salud-enfermedad, desde el momento que ingresa y durante el tiempo que conlleva el tratamiento, a través de la intervención directa con los usuarios y los factores influyentes 
del mismo como: su ámbito social y familiar, que permitan detectar, investigar, identificar, analizar las necesidades y las situaciones de riesgo social para los individuos, con la finalidad de garantizar y orientar al bienestar integral del paciente y su entorno familiar.

El Trabajador Social con los pacientes que reciben tratamiento sustitutivo de la función renal (diálisis) implementa programas, proyectos de prevención y detección temprana de la enfermedad renal, enfocados a los usuarios y sus familias, que garanticen el acceso a una salud de calidad y calidez durante todo el proceso que conlleva el tratamiento; desarrollando trans-diálisis varias actividades lúdicas que promueven la participación y entretenimiento de los adultos mayores, durante el tratamiento que dura de 4 a 5 horas.

El interés por llevar a cabo el presente artículo se debe a la importancia de la intervención del Trabajador Social en los diferentes campos de acción (Salud) con los usuarios, por el acceso a una Salud de Calidad y Calidez en las instituciones hospitalarias públicas y privadas.

El presente estudio aporta con datos cuantitativos, obtenidos producto de un proceso de intervención en el Departamento de Trabajo Social con los adultos mayores que recibían diálisis en un hospital público del Ecuador en el período octubre 2018 enero 2019. Con la aplicación de entrevistas enfocados al estado de salud y al entorno familiar-social de los pacientes por medio de un cuestionario y la ejecución de actividades lúdicas dentro del área de diálisis con los usuarios.

Producto de la búsqueda realizada se ubica que, existen pocos estudios de investigación sobre las funciones de Trabajo Social en atención a adultos mayores que reciben diálisis en las instituciones de salud sean públicas o privadas. Por lo cual el objetivo del presente artículo es dar a conocer el Rol de Trabajo Social en atención a adultos mayores que reciben diálisis, como un aporte al análisis de la intervención de Trabajo Social en este importante campo ocupacional.

\section{MATERIAL Y MÉTODOS}

El presente estudio de investigación es de tipo descriptivo-cuantitativo, en una población estudiada que fue de 14 adultos mayores que recibían tratamiento sustitutivo de la función renal (área de diálisis) en un Hospital Público del Ecuador, permitiendo recolectar información necesaria para la investigación, sustentada en estudios estadísticos que se 
evidencian en números, cantidades y variables, generando datos importantes y confiables de la población trabajada.

\section{Tal como plantea Cerón (2006):}

Es una estrategia de investigación que conceptualmente delimita propiedades de sus sujetos de estudio con el fin de asignarles números a las magnitudes, grados o tipos en que estos las poseen y que utiliza procedimientos estadísticos para resumir, manipular y asociar dichos números. Adicionalmente, con el fin de obtener la información necesaria para este trabajo, la investigación cuantitativa tiende a utilizar, aunque no necesariamente, la encuesta social y los muestreos probabilísticos. (pág. 38)

La investigación descriptiva permitió el análisis de datos sobre el tema de estudio de una manera efectiva y la identificación de las características de los sujetos de estudio, con la finalidad de analizar su situación y entorno socio-familiar, así como el Rol de Trabajo Social en atención a este tipo de pacientes que reciben tratamiento sustitutivo de la función renal, con hemodiálisis trisemanal.

Como expresa Arias (2012):

La investigación descriptiva consiste en la caracterización de un hecho, fenómeno, individuo o grupo, con el fin de establecer su estructura o comportamiento. Los resultados de este tipo de investigación se ubican en un nivel intermedio en cuanto a la profundidad de los conocimientos se refiere. (pág. 24)

Conforme a los fundamentos de la metodología científica, en el presente estudio se tomaron en cuenta como:

Criterios de inclusión: que sean adultos mayores con Insuficiencia Renal Crónica Terminal, que reciban hemodiálisis trisemanal, que asistan regularmente a su tratamiento en la unidad renal del Hospital Público en el cual se realizó el estudio y que expresen voluntariamente su deseo de participar en el estudio.

Criterios de exclusión: todos los criterios contrarios a lo manifestado como criterios de inclusión, que no facilitaron la recolección de información para el estudio planteado; así como también el que no podían participar debido a la condición física que presentaban y que motivó su hospitalización, impidiéndole participar en la entrevista. Se encontraban 
ausentes debido a la atención que recibían en las diferentes especialidades o por el tema de movilidad ya que vivián solos/as.

\section{Los instrumentos aplicados fueron:}

- El formulario de datos sociodemográficos constituido de 9 ítems: sexo, edad, estado civil, ciudad de residencia, zona de residencia, disponibilidad de vivienda, número de hijos, persona con la que acude al tratamiento y con quien vive.

El cuestionario sobre el estado de salud estuvo conformado por 3 ítems con opción de respuesta "si" o "no", ante preguntas sobre: la realización de ejercicios que son muy importantes para la salud de todas las personas en especial de los adultos mayores que padecen de insuficiencia renal, atención externa que hace referencia a la asistencia de especialidades a otras instituciones de Salud, atención complementaria que comprende las diferentes áreas: psicología, nutrición y Trabajo Social y 1 pregunta que corresponde a que tiempo recibe el tratamiento de diálisis.

La recolección de datos se realizó a través de la intervención de Trabajo Social con los adultos mayores que recibían diálisis en un hospital público del Ecuador, mediante la realización de entrevistas directas in situ, durante el tratamiento de diálisis que tenía una duración de 4 a 5 horas. El contenido de las entrevistas fue: sobre el estado de saludenfermedad y la relación socio-familiar de los usuarios.

El análisis de los datos, se efectuó por medio de la hoja de cálculo Microsoft Excel, que posibilitó el procesamiento de datos estadísticos para la obtención tanto de los porcentajes, promedios, así como la representación gráfica de la información obtenida con la aplicación del instrumento.

El aspecto ético fue muy importante la utilización de los diferentes principios en todo el proceso, como: principio de respeto, que hace referencia al momento de dirigirnos hacia el sujeto de estudio como fue la población adulta mayor con insuficiencia renal, mismo que permitió conocer el entorno socio-familiar en la cual se desarrollan, el principio de justicia desde el enfoque integral que recibían los pacientes en el proceso saludenfermedad y el principio de la confidencialidad que se guardó y se mantuvo durante la recolección de datos estadísticos y la tabulación de la información adquirida de la investigación. 


\section{RESULTADOS}

Se utilizaron los cuestionarios de datos sociodemográficos y del estado de salud a 14 adultos mayores que recibían diálisis durante el proceso académico relacionados al Trabajo Social en el área de salud de un hospital público del Ecuador.

Para la presente investigación se utilizó la hoja de cálculo Microsoft Excel, que facilitó a la obtención de los porcentajes, promedios, procesamiento de datos estadísticos, así como la representación gráfica de la información adquirida del instrumento aplicado, mismos que se da a conocer a continuación en dos aparatos:

La primera que corresponde a los datos sociodemográficos de los adultos mayores que recibían diálisis con los análisis cuantitativos y cualitativos respectivos:

Figura 1. Población según sexo.

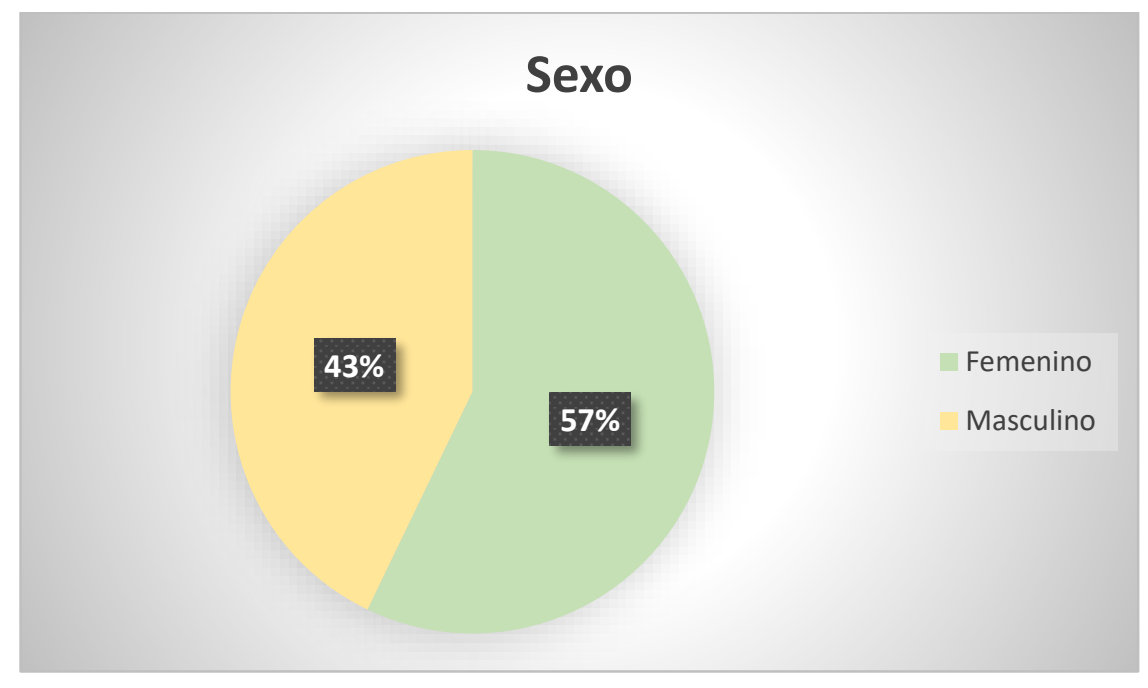

Elaborado por: Equipo de Investigación, (2020).

Análisis Cuantitativo: A través de la aplicación del cuestionario a la población total de 14 adultos mayores atendidos en el Hospital público del Ecuador que reciben diálisis, se evidencia que: el $57 \%$ de la población total que representa a 8 personas del sexo femenino y el $43 \%$ que representa a 6 personas del sexo masculino.

Análisis Cualitativo: En la presente investigación, se observó que existe una mayor incidencia de la enfermedad en las personas de la tercera edad del sexo femenino que asisten a recibir el tratamiento de diálisis en el Hospital público del Ecuador, mismas que se encuentran registradas en el Departamento de Trabajo Social y reciben la intervención de la Trabajadora Social de la Institución de Salud. 
Figura 2. Población según edad.

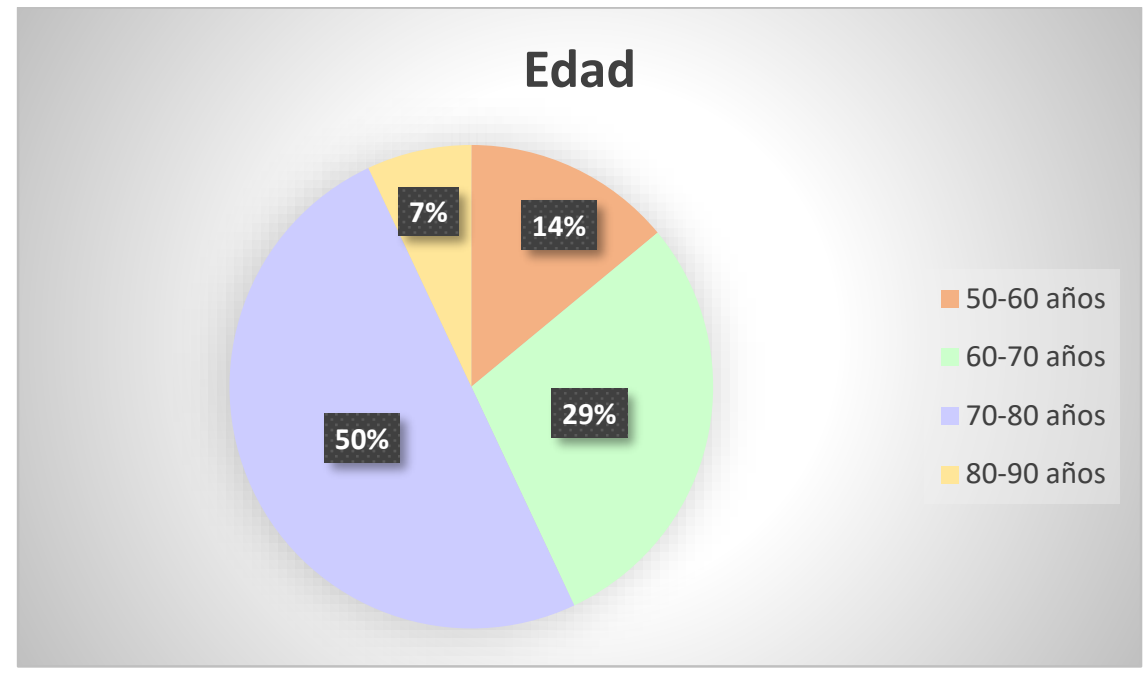

Elaborado por: Equipo de Investigación, (2020).

Análisis Cuantitativo: En el presente gráfico se refleja que: en el Hospital público del Ecuador la mayor parte de pacientes renales corresponden a la edad de adultos mayores, donde el $50 \%$ representa que van entre los 70 a 80 años de edad, seguido con el $29 \%$ que hace referencia a la edad entre los 60 a 70 años, sucesivo a esta el 14\% que corresponde entre los 50 a 60 años de edad y finalmente el 7\% que representa entre los 80 a 90 años de edad.

Análisis Cualitativo: Referente a los rangos de edad, se observa que la mayoría de la población total son adultos mayores que van entre los 70 a 80 años de edad entre el sexo masculino y femenino, que reciben diálisis, en el Hospital público del Ecuador, razón por cual se considera importante la intervención de Trabajo Social, ya que permitió cumplir con el principio de derecho al acceso de una salud de calidad y calidez.

Figura 3. Población según estado civil.

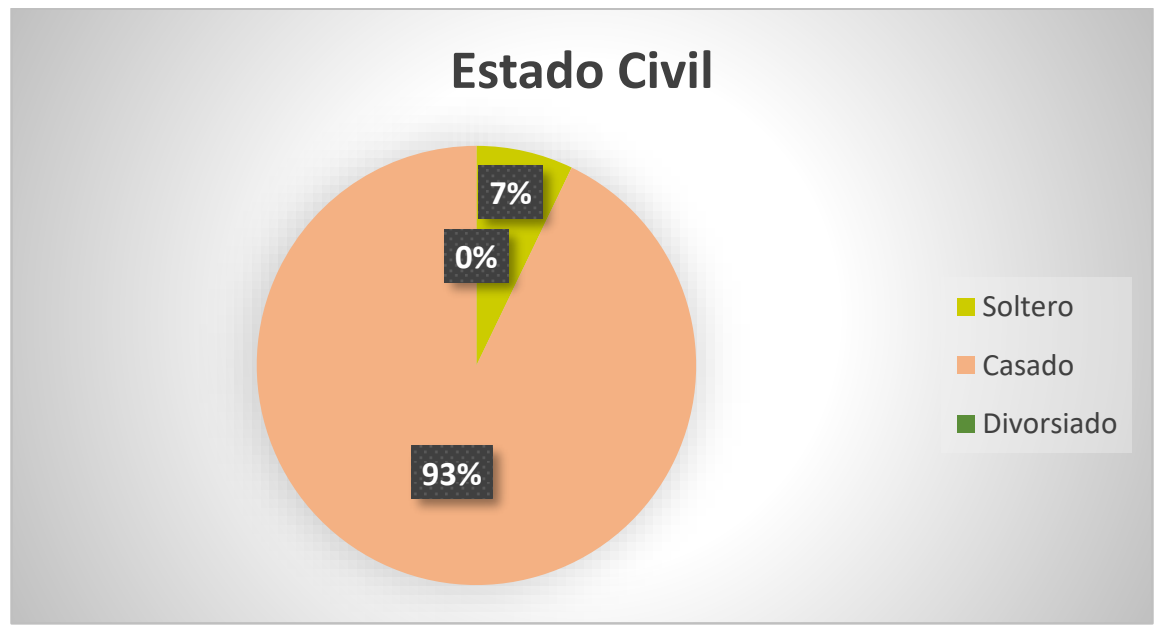

Elaborado por: Equipo de Investigación, (2020). 
Análisis Cuantitativo: En el Hospital público del Ecuador donde se realizó la investigación, en su mayoría las personas de la tercera edad que reciben diálisis son casados con el $93 \%$ lo que equivale a 13 adultos mayores, el $7 \%$ pertenece a 1 adulto mayor de estado civil soltero y ninguna de las personas en estudio son divorciados.

Análisis Cualitativo: Con respecto al estado civil de los pacientes que reciben diálisis, los resultados de la investigación, indican que la mayoría de la población en estudio se encuentran casados, lo que permitió a la Trabajadora Social conocer las redes familiares que poseen, e intervenir de manera directa y objetiva, por la importancia del apoyo familiar requerido en el tratamiento de la enfermedad renal, ya que son el núcleo principal de una persona, para que se lleve a cabo de una manera adecuada el proceso de recuperación.

Figura 4. Población según ciudad de residencia.

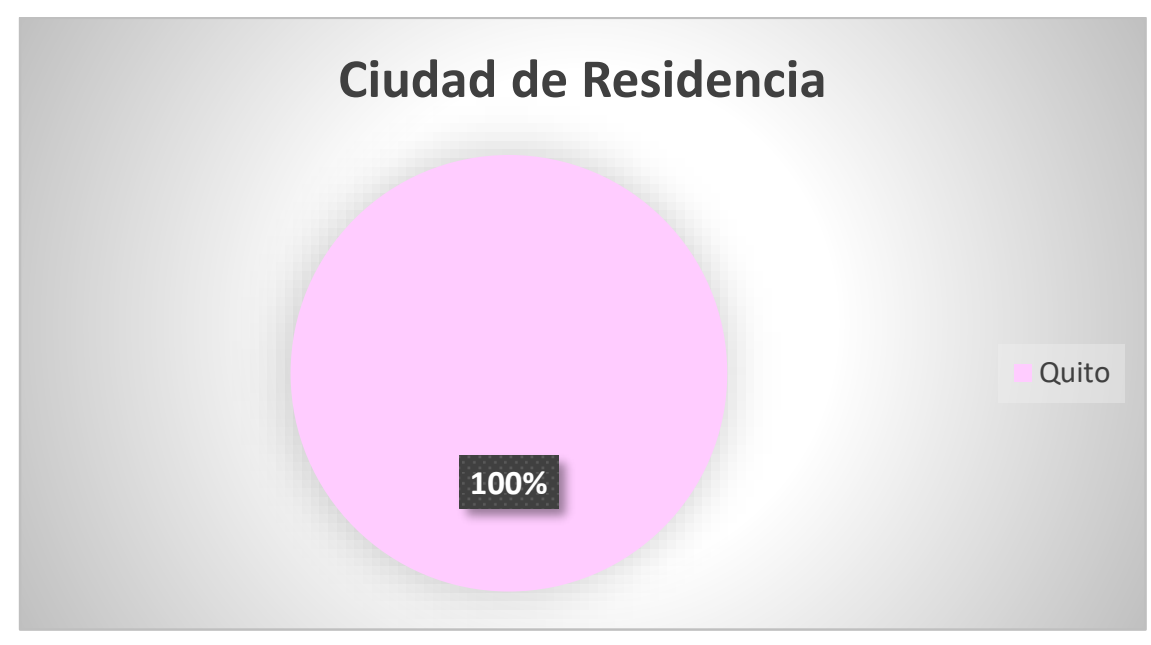

Elaborado por: Equipo de Investigación, (2020).

Análisis Cuantitativo: El presente gráfico representa la ciudad de residencia de los adultos mayores que reciben diálisis en el Hospital público del Ecuador. Efectivamente, los resultados de la investigación realizada, evidencian que el 100\% de los adultos mayores son de la Ciudad de Quito de la Provincia de Pichincha.

Análisis Cualitativo: En la presente investigación, se evidenció que todos los adultos mayores que reciben diálisis en el Hospital público del Ecuador son residentes de la ciudad de Quito, en sectores como el: norte, centro y sur, lo que es importante para asistir al tratamiento de diálisis que tiene una duración de 4 a 5 horas, en forma trisemanal, y a los chequeos médicos de las diferentes especialidades que son complementarias para el tratamiento sustitutivo de la función renal. 
Figura 5. Población según zona de residencia.

\section{Zona de Residencia}

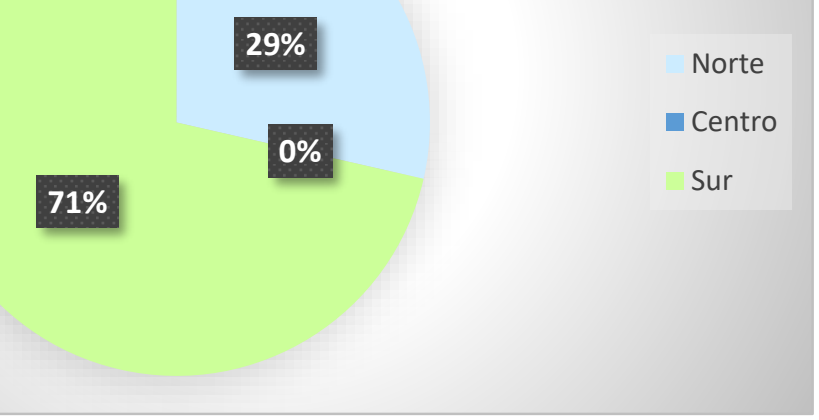

Elaborado por: Equipo de Investigación, (2020).

Análisis Cuantitativo: De la población total de 14 adultos mayores. El $71 \%$ representa a las 10 personas viven en la zona sur de la Ciudad de Quito, seguido del 29\% que refleja a los 4 adultos mayores que viven en la zona norte de la Ciudad de Quito.

Análisis Cualitativo: Con respecto a la zona de residencia de los adultos mayores que reciben diálisis en el Hospital público del Ecuador, se evidenció que la gran parte de los pacientes habitaban al sur de la ciudad, lo que en ocasiones provocaba dificultades de movilización para acudir a los tratamientos y a la atención de algunas especialidades dentro de la Institución de Salud.

Figura 6. Población según disponibilidad de vivienda.

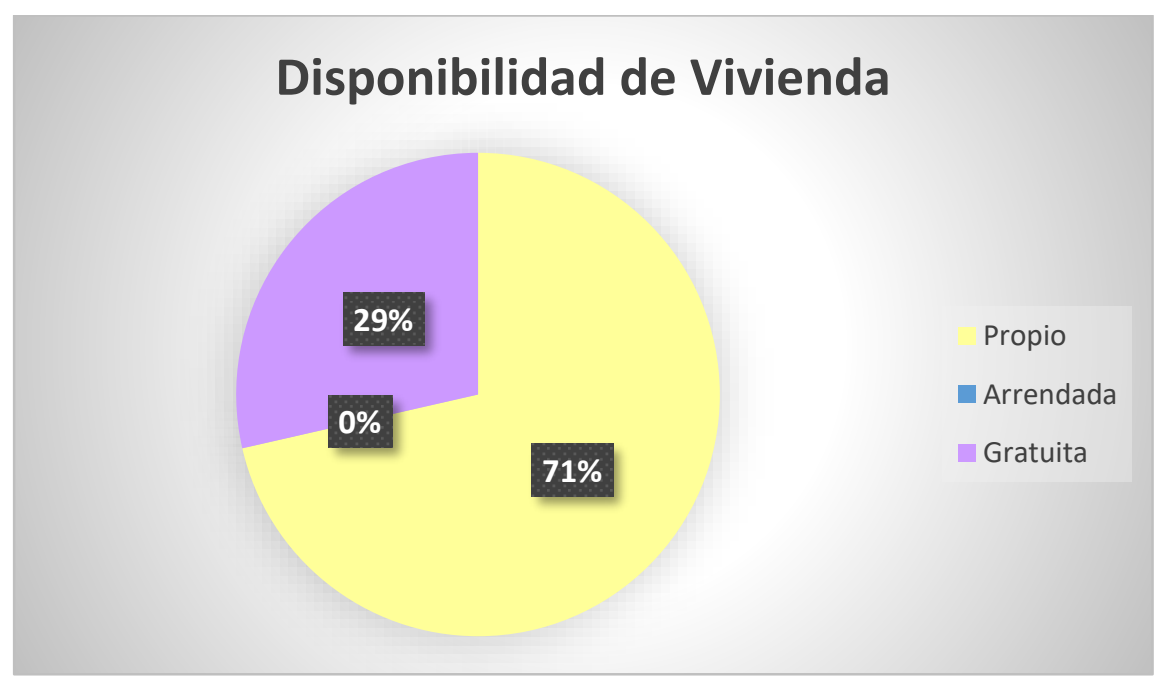

Elaborado por: Equipo de Investigación, (2020). 
Análisis Cuantitativo: La aplicación del cuestionario indicado, a los adultos mayores que reciben diálisis en el Hospital público del Ecuador, reflejó que el $71 \%$ hace énfasis a los 10 adultos mayores que cuentan con una vivienda propia, seguido del $29 \%$ correspondiente a los 4 adultos mayores que tienen una casa que es gratuita y ninguno de los usuarios cuentan con una vivienda arrendada.

Análisis Cualitativo: Con respecto a la disponibilidad de la vivienda, es un componente muy importante, ya que permite ver la relación de ingresos y egresos de los usuarios. Tanto así que, se obtuvo una mayoría de adultos mayores que reciben diálisis en el Hospital público del Ecuador que poseen una vivienda propia, realidad que les brinda seguridad para que lleven a cabo las actividades cotidianas junto a su entorno familiar, y ello consecuentemente genera un estado emocional equilibrado, una autoestima sólida y mayor seguridad para realizar el tratamiento de diálisis.

Figura 7. Población según número de hijos.

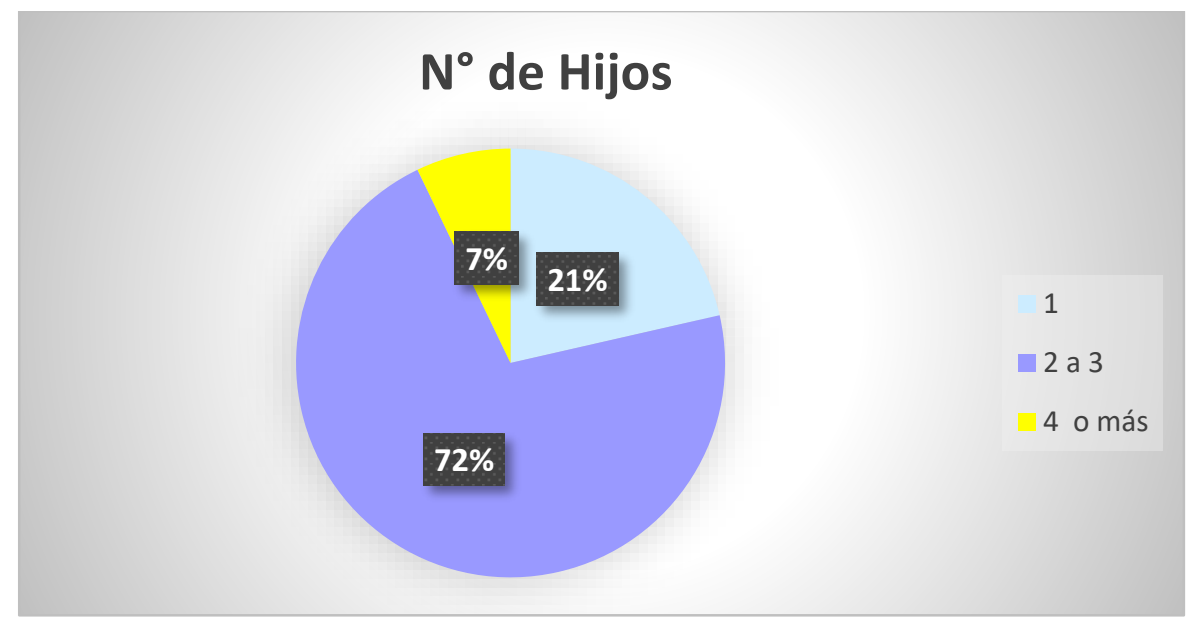

Elaborado por: Equipo de Investigación, (2020).

Análisis Cuantitativo: Con respecto al número de hijos de las personas de la tercera edad que reciben diálisis en el Hospital público del Ecuador, producto de la investigación se obtiene que: el $72 \%$ de los entrevistados (10 usuarios) tienen de 2 a 3 hijos, el $21 \%$ (3 pacientes) que tienen 1 hijo, y el 7\% que hace referencia (1 adulto mayor) que tiene más de 4 hijos.

Análisis Cualitativo: En la presente investigación realizada a los adultos mayores que reciben diálisis en la Institución de Salud, se obtuvo que la mayor parte de pacientes renales tienen entre 2 a 3 hijos, entendiendo que son el entorno familiar más próximo que brinda la atención necesaria y la estabilidad necesaria durante el tiempo que perdure el 
tratamiento (toda la vida), ya que esta enfermedad requiere de mucha atención por los cambios que provoca tanto en actividades de la vida cotidiana como en su estado emocional.

Figura 8. Población según persona que les acompaña a la diálisis.

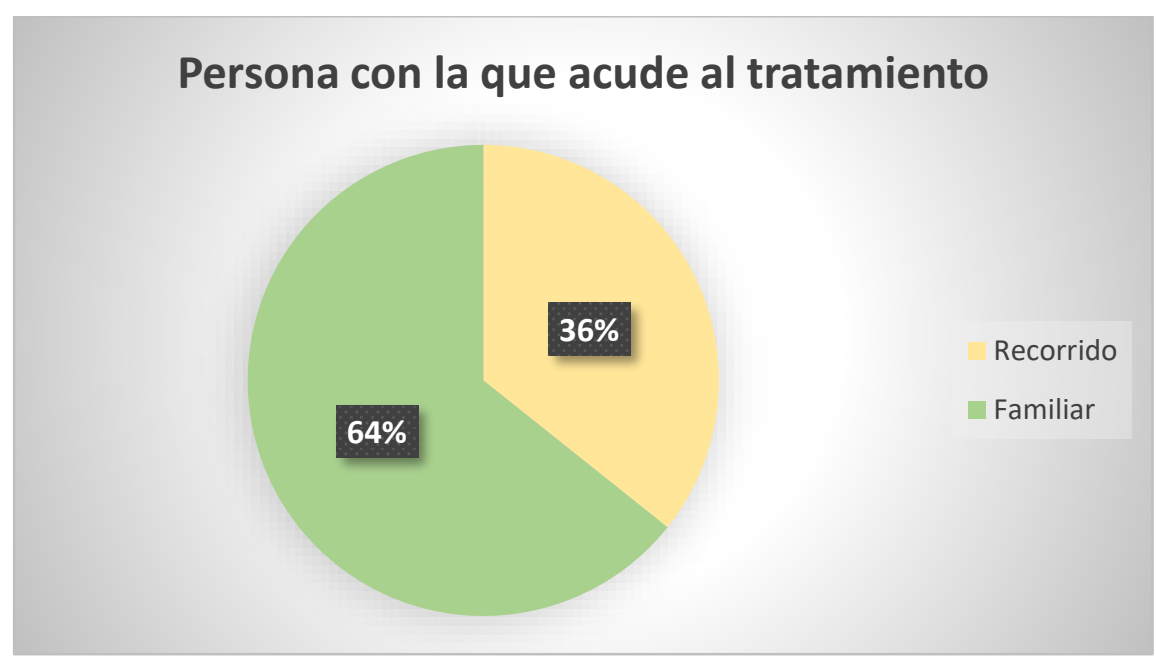

Elaborado por: Equipo de Investigación, (2020).

Análisis Cuantitativo: El 64\% de la población total de los adultos mayores que reciben diálisis, acuden al tratamiento acompañado de un familiar, mientras que el 36\% de los encuestados, asisten al tratamiento en el recorrido - transporte que brinda la Institución de Salud (puerta a puerta).

Análisis Cualitativo: Con respecto a la persona con quien acuden los adultos mayores a la diálisis, indicaron que lo hacen acompañados de un familiar, lo cual les genera seguridad, confianza y les brinda el apoyo en el proceso que conlleva la enfermedad y sobre todo porque la familia es el núcleo fundamental para la recuperación de este tipo de pacientes.

Figura 9. Población según persona con quien vive. 


\section{Con quién vive?}

\section{$7 \%$}

Familiar

Solo

Análisis Cuantit Elaborado por: Equipo de Investigación, (2020). $\quad$ lecir el 93\% que corresponde (13 adultos mayores) viven con sus familiares, y apenas el $7 \%$ de los pacientes viven solos.

Análisis Cualitativo: Los resultados obtenidos, evidencian que la mayoría viven con sus familiares directos (esposo/a, hijos/as, y sus hermanos/as), que son una base fundamental para el tratamiento de la enfermedad renal, brindan el soporte emocional y económico necesario que permita respaldar el tratamiento sustitutivo de la función renal.

De manera concomitante, los resultados obtenidos de la aplicación del cuestionario sobre el estado de salud de las personas de la tercera edad, se detallan a continuación, desde una perspectiva cualitativa y cuantitativa:

Figura 10. Población según actividad física.

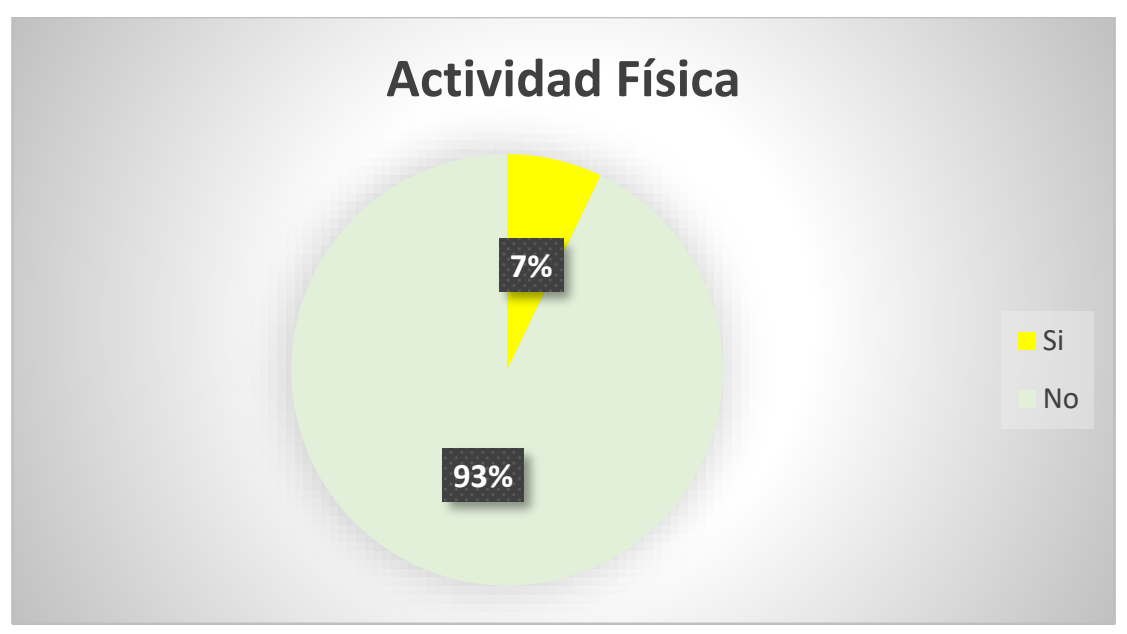

Elaborado por: Equipo de Investigación, (2020).

Análisis Cuantitativo: El 93\% de la población de los adultos mayores encuestados que reciben diálisis indican no realizar actividad física, y apenas el 7\% de adultos mayores señalan que si realizan ejercicios. 
Análisis Cualitativo: Con respecto a este item, la mayoría de las personas de la tercera edad encuestadas, no realizan actividades físicas, debido a las condiciones personales y familiares, ya que no todos cuentan con un acompañante para realizar los ejercicios y algunas tienen algún tipo de discapacidad física, sin embargo la actividad física es muy importante para todas las personas en lo particular para los adultos mayores que reciben diálisis, puesto que por su avanzada edad, surgen muchos inconvenientes relacionados con la salud, razón por la cual el realizar ejercicios ayuda al bienestar físico, emocional del paciente, y por ende les permite promover su calidad de vida.

Figura 11. Población según quienes reciben atención externa

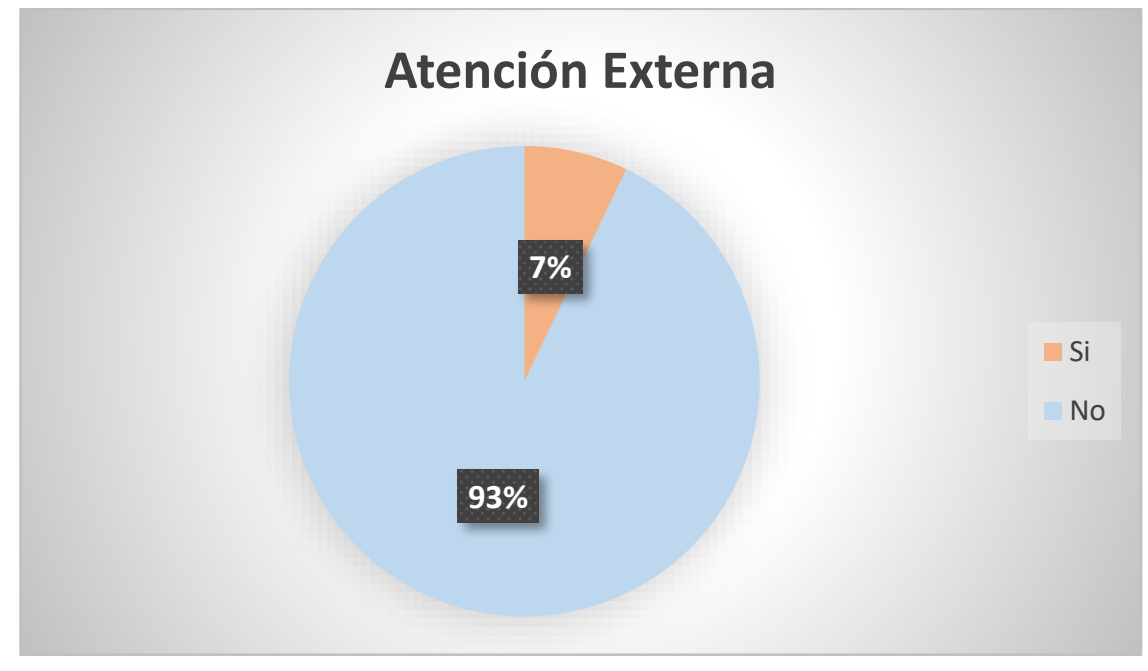

Elaborado por: Equipo de Investigación, (2020).

Análisis Cuantitativo: En el presente gráfico, se puede visualizar que los adultos mayores que reciben diálisis en el Hospital público del Ecuador, no reciben atención externa que corresponde a 13 adultos mayores correspondientes al 93\% de las personas de la población total, siendo apenas el 7\% (1 paciente) que si asiste a consultas externas a la Institución de Salud.

Análisis Cualitativo: En la investigación realizada a las personas de la tercera edad que reciben diálisis, se observó que la mayor parte no reciben atención médica externa a la Institución de Salud, ya que muchos usuarios posiblemente no ven necesario realizarse chequeos fuera, debido a la atención que reciben de todas las especialidades complementarias de la enfermedad en el hospital.

Figura 12. Población según quienes reciben atención complementaria. 


\section{Atención complementaria}

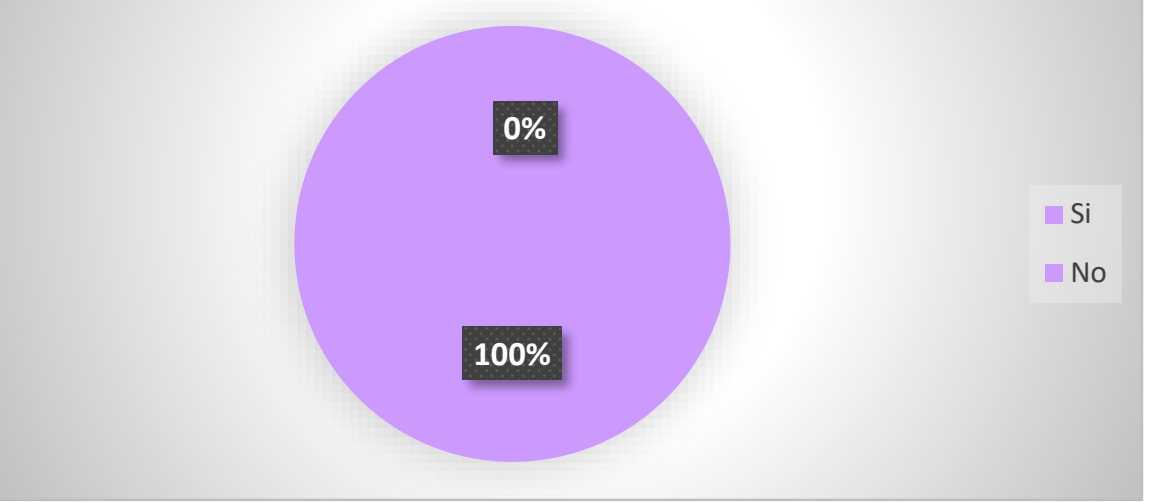

Elaborado por: Equipo de Investigación, (2020).

Análisis Cuantitativo: De la población total de 14 adultos mayores que reciben diálisis, se evidenció que todos (el 100\%), reciben atención complementaria.

Análisis Cualitativo: En la investigación realizada a la población de estudio, se pudo observar que todos los pacientes renales si recibían atención (nutrición, psicología y Trabajo Social), mismas que fueron muy importantes para que los usuarios tengan una soporte complementario, oportuna.

Figura 13. Población según tiempo que recibe diálisis.

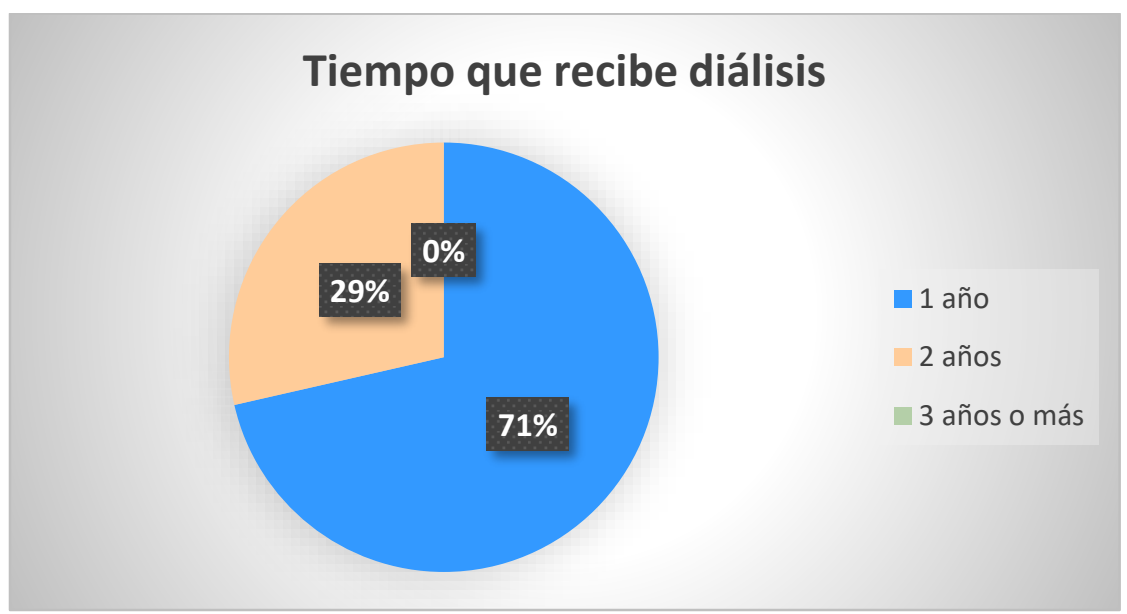

Elaborado por: Equipo de Investigación, (2020).

Análisis Cuantitativo: El 71\% de la población total de 14 adultos mayores investigados, refieren que reciben diálisis durante 1 año, seguido el 29\% (4 usuarios) que reciben diálisis 2 años.

Análisis Cualitativo: Con relación al tiempo que reciben diálisis las personas investigadas, se evidenció que la mayoría de los adultos mayores reciben el tratamiento 
sustitutivo de la función renal (diálisis) durante 1 año en la Institución de Salud y, se encuentran registrados en el Departamento de Trabajo Social, recibiendo la orientación complementaria necesaria, según cada caso.

\section{DISCUSIÓN}

En la presente investigación, es necesario considerar datos como la atención de Trabajo Social a las personas que reciben diálisis en las Instituciones de Salud como enfatizan en la investigación de Macías, Cedeño y Menéndez (2018):

La Intervención de las Trabajadoras Sociales con los pacientes que reciben diálisis se da de manera permanente desde el momento de su ingreso hasta el total de la duración de su tratamiento, con el objetivo de brindar la atención social integral a los involucrados directos y sus familiares, empezando por el estudio de las necesidades de las personas para que a través de los recursos disponibles se fomente el bienestar social. (p. 13)

El Trabajador Social con los usuarios que reciben diálisis, interviene de forma directa desde la primera instancia y durante el tiempo que conlleva el tratamiento de la función renal (diálisis), con la finalidad de garantizar el bienestar integral en la salud, cuidado, atención, tratamiento y seguimiento de los pacientes, que se realiza de manera continua. Otro aspecto a destacar son los Roles de Trabajo Social en Atención a pacientes que reciben Diálisis, que es muy amplio y esencial, ya que la intervención se realiza desde el primer momento que el paciente ingresa a la Institución de Salud y el tiempo que perdure el tratamiento, garantizando el desarrollo y bienestar integral del individuo, a través de la aplicación de las diferentes metodologías, modelos, técnicas e instrumentos propios de la profesión para una intervención efectiva con la colaboración de los usuarios y el entorno socio-familiar, como muestra en el estudio realizado por Macías, Cedeño y Menéndez (2018):

Tabla 1. Roles y actividades de Trabajo Social en Atención a Pacientes que reciben Diálisis. 


\begin{tabular}{|c|c|c|}
\hline Roles & Actividades & Instrumentos \\
\hline Asistencial & $\begin{array}{l}\text { - Acogida del paciente, registro de datos personales y del caso. } \\
\text { - Registro paulatino de las intervenciones con cada uno de los } \\
\text { casos. } \\
\text { - Acompañamiento social } \\
\text { - Consulta externa de Trabajo Social }\end{array}$ & $\begin{array}{l}\text { - Ficha social } \\
\text { - Historia clínica } \\
\text { - Historia Socia }\end{array}$ \\
\hline Investigador & $\begin{array}{l}\text { - Estudio global de las situaciones para identificar las dificultades, } \\
\text { factores causales limitantes o facilitadores en el contexto del } \\
\text { paciente. } \\
\text { - Visitas domiciliarias } \\
\text { - Elaboración de informe social } \\
\text { - Participar en la elaboración del diagnóstico de salud }\end{array}$ & $\begin{array}{l}\text { - Entrevista } \\
\text { - Cuaderno de notas } \\
\text { - Informe Social } \\
\text { - Diagnostico Social } \\
\text { - Escalas de valoración }\end{array}$ \\
\hline Orientador & $\begin{array}{l}\text { - Orientación y asesoramiento acerca del tratamiento } \\
\text { - Orientación de los factores de riesgo para el enfermo renal } \\
\text { - Elabora y ejecuta junto al equipo interdisciplinario charlas de } \\
\text { hábitos saludables }\end{array}$ & - Material de difusión \\
\hline Gestionador & $\begin{array}{l}\text { - Tramites de derivación intra y extra institucionales } \\
\text { - Coordina la atención de los pacientes con otras instituciones de } \\
\text { acuerdo a los convenios }\end{array}$ & $\begin{array}{l}\cdot \text { Hojas de derivación } \\
\cdot \text { Hojas de interconsulta } \\
\cdot \text { Documentación del } \\
\text { paciente }\end{array}$ \\
\hline
\end{tabular}

Nota: La tabla muestra los roles, actividades e instrumentos de Trabajo Social en Atención a Pacientes que reciben Diálisis.

Fuente: Tomado de Macías, et al (2018, pág. 12). El Trabajo Social Sanitario: Un Análisis en los Centros de Diálisis del Cantón Portoviejo.

El profesional en este campo debe intervenir desde el enfoque de derechos, garantizando

el cumplimiento al acceso del servicio de salud de calidad y calidez que deben recibir todas las personas, sobre todo los adultos mayores que padecen de insuficiencia renal, debido a que surgen cambios en el aspecto físico como: cansancio, fatiga, dolor, dificultades para respirar al movilizarse y otros aspectos que afectan al metabolismo por la alimentación adecuada que deben ingerir y por los medicamentos que pueden ocasionar nauseas, dolor de cabeza, mismos que dificultan al proceso de recuperación de los pacientes renales.

Otra investigación realizada por (Giler, 2019) sobre pacientes renales y su caracterización como aporte al proceso de intervención del Trabajo Social indica acerca de los roles del Trabajador Social con pacientes renales en el área de diálisis que son diversos y que se debe partir desde las necesidades y los requerimientos que soliciten los pacientes para una intervención segura, que permitan la detección de los factores de riesgo a los cuales están expuestos los pacientes que reciben el tratamiento sustitutivo de la función renal (diálisis) tanto en el aspecto familiar, social e institucional:

Tabla 2. Roles que debe realizar el profesional de Trabajo Social con los pacientes renales Roles que debe realizar el profesional de Trabajo Social 


\begin{tabular}{|c|c|}
\hline Investigación & $\begin{array}{l}\text { Determinar los factores de riesgos que afecten al usuario mediante la } \\
\text { investigación social, así como mantener datos e información actualizada } \\
\text { para atender oferta y demanda de servicios. En este punto se hace hincapié } \\
\text { en el trabajo interdisciplinario que existe entre los departamentos de } \\
\text { Psicología, Nutrición y Enfermería. }\end{array}$ \\
\hline $\begin{array}{l}\text { Prevención y } \\
\text { recuperación }\end{array}$ & $\begin{array}{l}\text { El Trabajador social fortalecerá vínculos que permitan la unidad familiar } \\
\text { en beneficio del paciente, fomentando la participación en su recuperación } \\
\text { y tratamiento. Una vez identificados factores de riesgo, el trabajador } \\
\text { social diseña las acciones que eviten el deterioro de la salud del paciente } \\
\text { y mejoren su calidad de vida. }\end{array}$ \\
\hline $\begin{array}{l}\text { Problemas que } \\
\text { identifican los } \\
\text { trabajadores } \\
\text { sociales en este tipo } \\
\text { de atención }\end{array}$ & $\begin{array}{l}\text { Se conoce que existen pacientes con Familiares conflictivos, mismos que } \\
\text { a pesar de la labor que el departamento realiza exigen bonos, siendo un } \\
\text { tema que no es manejado a través de esta área y que generalmente se les } \\
\text { explica en la inducción. }\end{array}$ \\
\hline $\begin{array}{l}\text { Programas, } \\
\text { proyectos } \\
\text { acciones } \\
\text { ejecuta el trabajo } \\
\text { social con pacientes } \\
\text { renales }\end{array}$ & $\begin{array}{l}\text { Debe tomarse en cuenta la disponibilidad del grupo para poder llevar a } \\
\text { cabo actividades de índole socioeducativa, sea estos talleres, charlas, } \\
\text { información acerca de la Unidad de técnica. El área de trabajo social } \\
\text { depende de otras personas para operar en beneficio del paciente, pero se } \\
\text { ve mermado por la disponibilidad de recursos, tanto para la recolección } \\
\text { de datos y desarrollar intervenciones a profundidad con el paciente y la } \\
\text { familia, como para la movilización, misma que debe ajustarse a la } \\
\text { programación que el profesional realice en base a sus demás actividades. }\end{array}$ \\
\hline
\end{tabular}

Nota: La tabla muestra los roles que debe realizar Trabajo Social con los pacientes renales

Fuente: Tomado de Giler (2019, pp. 46-48). Pacientes renales y su caracterización como aporte al proceso interventivo del Trabajo Social. Caso de una clínica de diálisis en la ciudad de Guayaquil.

El Rol de Trabajo Social con los pacientes que reciben diálisis, es muy importante, debido a que el profesional forma parte del equipo multidisciplinario y realiza la intervención percibiendo a los usuarios como sujetos de derechos en el proceso de salud-enfermedad, donde se debe trabajar de forma directa con el paciente y su familia, que son el núcleo primordial al momento de ser diagnosticada la enfermedad renal, para la aceptación del nuevo estilo de vida que deben enfrentar durante toda la vida y el proceso de recuperación, 
ya que es el entorno que forma parte del vínculo de afectividad, comunicación, apoyo y seguridad para el proceso de una recuperación adecuada que deben tener los pacientes renales.

El Trabajador Social debe intervenir con los adultos mayores que reciben diálisis, como promotor del cumplimiento de los derechos y deberes que tienen los pacientes, sobre todo a la población adulta mayor por su mayor grado de vulnerabilidad que presenta en la sociedad, y debe implementar programas y proyectos enfocados a la prevención, participación de los individuos y sus familiares, detección temprana de la enfermedad, mismos que permitan descubrir las posibles problemáticas, necesidades y riesgos sociales a los cuales se encuentran expuestos.

Además, el profesional desde el punto de vista ético, en esta área utiliza el principio de la confidencialidad, el respeto, la comunicación asertiva, debido a que permite entender y comprender la dinámica social en la que se encuentran inmersas las persona de la tercera edad que reciben el tratamiento sustitutivo de la función renal (diálisis), por ello es necesario dar a conocer los diferentes servicios y recursos que presta la Institución de Salud y a los cuales pueden acceder mientras acuden al tratamiento de diálisis que tiene una duración de 4 a 5 horas, ya que muchos usuarios desconocen los requisitos necesarios para poder acceder a los mismos.

Los datos de la población con la enfermedad renal como muestra en el estudio realizado por la (Federación Internacional del Día Mundial del Riñón, 2019), acerca de la enfermedad renal, en el cual indica que es la sexta causa de muerte de más rápido crecimiento, en la que se estima que 850 millones de personas en el mundo padecen enfermedad renal por diversas causas.

En la investigación elaborada por la Sociedad Latinoamericana de Nefrología e Hipertensión, citado en (Ministerio de Salud Pública MSP, 2015), sobre el programa nacional de salud renal, indica que la prevalencia de la enfermedad renal en América latina es de 650 pacientes por cada millón de habitantes, con un incremento estimado del $10 \%$ anual. Donde el Ecuador tiene 16`278.844 habitantes, en el que se estima que para el año 2015 los pacientes con insuficiencia renal serán 11.460 personas.

Con respecto a la población adulta mayor, se evidencia datos importantes en el estudio de las (Naciones Unidas, s.f) sobre el envejecimiento, en la cual señala que para el 2050, una de cada seis personas en el mundo tendrá más de 65 años que equivale al 16\%, más que 
la proporción actual, de una de cada 11 en este 2019 que representa el 9\%. Se estima que el número de personas de 80 años o más se triplicará, de 143 millones en 2019 a 426 millones en 2050.

Asimismo, en el estudio realizado por (Jiménez \& Quezada, 2008), donde se indica que, en el año 2005 la población total de América Latina se cifraba en 546,7 millones de personas, de los que el 8,8\% contaban con 60 años y más. Las proyecciones apuntan un incremento notable de la población adulta mayor en el territorio latinoamericano, de forma que, para el año 2025, el 14,5\% de la población se situará ya en este grupo etario. A nivel nacional con respecto a las personas de la tercera edad, muestran datos del estudio elaborado por el (Instituto Nacional de Estadística y Censos INEC, 2009) sobre Salud, Bienestar y envejecimiento 2009, indica que, en el año 2009, se tenía mayor población de 60 años en la región Sierra y existe una diferencia porcentual mínima entre hombres y mujeres, que corresponde al $6.8 \%$.

Otra investigación, de la (Defensoría del Pueblo de Ecuador DPE, 2018) sobre la información estadística de casos referentes a personas adultas mayores llevados por la DPE, hace referencia, que existían más de 940.000 personas mayores de 65 años, que representan el 6,5\% de la población nacional, y para el 2017 se estimaba que serían 1.180.944, es decir el 7\% de la población. La distribución por género de la población adulta mayor a nivel nacional es de $52,61 \%$ para las mujeres y de $47,39 \%$ para los hombres.

La investigación y seguimiento del presente tema a nivel local, refleja datos como en el estudio del (Instituto Nacional de Estadística y Censos INEC, 2008) sobre la población adulta mayor en la ciudad de Quito, estudio de la situación sociodemográfica y socioeconómica, indica que la población total de la ciudad de Quito urbana es de 1.523.274 habitantes. Esta población está constituida por $746.923(49,0 \%)$ hombres y $776.352(51,0 \%)$ mujeres. Las personas de la tercera edad en la ciudad de Quito para ambos sexos son de 90.628 (5,9\%) con respecto a la población total de esta ciudad. Lo mismo sucede para adultos mayores hombres 40.967 (5,5\%) y para adultas mayores mujeres $49.660(6,4 \%)$.

Los datos sociodemográficos de la población adulta mayor con insuficiencia renal en lo que concierne a: sexo, edad, estado civil, ciudad de residencia, disponibilidad de vivienda, número de hijos, tiempo que reciben diálisis nos indica datos similares, estableciéndose 
una comparación con la investigación en el estudio realizado por (Merino, 2018), sobre estrategias de afrontamiento en pacientes con insuficiencia renal crónica hospitalizados en el área de nefrología del Hospital Carlos Andrade Marín, en el cual muestra que la edad promedio fue de 42-50 años de edad y comparativamente hablando en el presente estudio la edad promedio va entre 70-80 años, lo que señala que se trabajó con la población adulta mayor, en cuanto al sexo indica que la mayor parte son del sexo masculino que corresponde al $60 \%$ y en la investigación realizada muestra que son del sexo femenino que equivale al 57\%, con respecto al aparado del estado civil, se evidencia que en los dos estudios investigativos tienen el mismo comportamiento, con un porcentaje mayoritario del $93 \%$ y $50 \%$ de los adultos mayores que son casados, en lo que concierne a la variable de número de hijos los datos indican que tienen de 1-5 hijos y relacionado al presente estudio manifiestan que los adultos mayores poseen de 2 a 3 hijos, con relación a la vivienda los dos trabajos investigativos muestran que residen en la Ciudad de Quito, acerca del componente del tiempo que reciben el tratamiento los datos revela que va desde 1 mes a 6 meses a diferencia de la investigación realizada que reciben 1 año el tratamiento de diálisis las personas de la tercera edad.

En el presente estudio investigativo, otro de los datos relevantes hace referencia al estado de salud de los pacientes renales, como la actividad física que es muy importante para la salud de todas las personas, en especial de los adultos mayores que reciben tratamiento sustitutivo de la función renal (diálisis), debido a su avanzada edad surgen cambios tanto en su aspecto físico como en su vida cotidiana, y por la misma enfermedad surgen cambios en su estilo de vida ya que deben recibir el tratamiento de por vida, razón por la cual la actividad física es beneficio para la salud de los pacientes, como plantea la Organización Mundial de la Salud (2020):

La OMS define la actividad física como cualquier movimiento corporal producido por los músculos esqueléticos, con el consiguiente consumo de energía. Ello incluye las actividades realizadas al trabajar, jugar y viajar, las tareas domésticas y las actividades recreativas. La actividad física tanto moderada como intensa es beneficiosa para la salud.

Otra dimensión con respecto al estado de salud, es la atención complementaria que está compuesta por diferentes áreas: psicología, nutrición y Trabajo Social que deben recibir los adultos mayores con insuficiencia renal, mismas que permiten llevar a cabo el 
tratamiento de una manera adecuada y equilibrada en el proceso de salud-enfermedad de los pacientes.

\section{CONCLUSIONES}

- El presente estudio estableció que el Rol de Trabajo Social en atención a adultos mayores que reciben diálisis es de especial relevancia, por la intervención realizada y los resultados obtenidos de las actividades desarrolladas. Con ello se cumple el objetivo planteado. Describir el Rol de Trabajo Social con las personas de la tercera edad que reciben el tratamiento sustitutivo de la función renal (diálisis).

- El Rol de Trabajo Social con los adultos mayores que reciben diálisis, se debe realizar de manera directa con el paciente y el entorno socio-familiar en la cual se desarrollan, desde el primer momento y durante el tiempo que persista el tratamiento, garantizando el acceso a una salud de calidad y calidez que deben recibir los usuarios en la Institución de Salud.

- El Trabajador Social con los pacientes que reciben diálisis, realiza la intervención desde las necesidades y las problemáticas que presentan los usuarios durante el tratamiento, a través de la aplicación de las metodologías, modelos, técnicas e instrumentos, que permiten tener una intervención efectiva en cada caso correspondiente.

- El objetivo del profesional con las personas de la tercera edad que reciben tratamiento sustitutivo de la función renal (diálisis), es garantizar el bienestar integral del individuo, cumplimiento de los derechos, orientar a la resolución de problemas y el desarrollo de las habilidades y potencialidades de los pacientes durante el tratamiento, mismo que tiene una duración de 4 a 5 horas.

- A través de la implementación y aplicación de los instrumentos, se ha obtenido de datos sociodemográficos como: sexo, edad, estado civil, ciudad de residencia, zona de residencia, disponibilidad de vivienda, número de hijos, persona con la que acude al tratamiento, con quien vive, e información sobre el estado de salud de la población adulta mayor que recibían diálisis.

- La intervención de Trabajo Social en el área de diálisis, permite desarrollar nuevos enfoques desde las diferentes realidades que atraviesan los adultos mayores por su mayor grado de vulnerabilidad, ya que la enfermedad renal produce cambios a nivel individual, familiar, social como las complicaciones para la alimentación, la ingesta 
de medicamentos, las actividades cotidianas, mismas puede provocar que la familia y el entorno en el que se desarrolla lo excluya o lo rechace.

- La intervención de la Trabajadora Social promueve la facilitación de los procesos familiares, a fin de que la población adulta mayor cuente con el debido apoyo familiar como soporte anímico - emocional en el tratamiento sustitutivo de la función renal.

- A través de las actividades desarrolladas por la población investigada, se observó el reconocimiento de las habilidades y potencialidades que poseían los adultos mayores con insuficiencia renal y su receptividad para la participación de los compañeros/as en las diferentes actividades ejecutadas.

\section{REFERENCIAS BIBLIOGRÁFICAS}

Arias, F. G. (2012). El Proyecto de Investigación. Introducción a la Metodología Científica (Vol. $6^{\text {a }}$ Edición). Editorial Episteme. Obtenido de https://ebevidencia.com/wp-content/uploads/2014/12/EL-PROYECTO-DEINVESTIGACI\%C3\%93N-6ta-Ed.-FIDIAS-G.-ARIAS.pdf

Ávila, G. J. (2020). Trabajo social, en salud: Teoría y praxis innovadora. Revista Margen de Trabajo Social y Ciencias Sociales No.97.

Cerón, M. C. (2006). Metodologías de la investigación social. LOM ediciones.

Defensoría del Pueblo de Ecuador DPE. (2018). Información estadística de casos referentes a personas adultas mayores llevados por la DPE. Quito: Dirección Nacional de Investigación e Incidencia en Política Pública. Obtenido de http://repositorio.dpe.gob.ec/bitstream/39000/2191/1/DEOI-DPE-012-2018.pdf

Federación Internacional de Trabajadores Sociales FITS. (2020). Definición Global del Trabajo Social. Obtenido de https://www.ifsw.org/what-is-social-work/globaldefinition-of-social-work/definicion-global-del-trabajo-social/

Federación Internacional del Día Mundial del Riñón. (14 de marzo de 2019). Salud renal para todos, en todas partes. Obtenido de Federación Internacional del Día Mundial del Riñón: https://www.worldkidneyday.org/wkd-2019-spanish/

Giler, V. J. (2019). Pacientes renales y su caracterización como aporte al proceso interventivo del Trabajo Social. Caso de una clínica de diálisis en la ciudad de Guayaquil. Trabajo de Titulación. Universidad Católica de Santiago de Guayaquil, Guayaquil, Ecuador. Obtenido de http://192.188.52.94/bitstream/3317/13809/1/T-UCSG-PRE-JUR-TSO-106.pdf 
Instituto Nacional de Estadística y Censos INEC. (2008). La población adulto mayor en la ciudad de Quito, estudio de la situación sociodemográfica y socioeconómica. Obtenido de Instituto Nacional de Estadística y Censos INEC: https://www.ecuadorencifras.gob.ec/documentos/web-

inec/Bibliotecas/Estudios/Estudios_Socio-

demograficos/Poblac_Adulto_Mayor.pdf

Instituto Nacional de Estadística y Censos INEC. (2009). Salud, Bienestar y envejecimiento. Obtenido de Instituto Nacional de Estadística y Censos INEC: https://www.ecuadorencifras.gob.ec/wpcontent/descargas/Presentaciones/estadisticas_adulto_mayor.pdf

Jiménez, A., \& Quezada, M. (2008). Extensión de la protección social en salud a poblaciones en condiciones especiales de vulnerabilidad (adulto mayor y dependencia). Consorcio EUROsociAL Salud. Obtenido de http://www.oiss.org/wp-content/uploads/2000/01/Doc_Adultos_MAyores.pdf

Macías, K., Cedeño, M., \& Menéndez, F. (2018). El trabajo social sanitario: un análisis en los centros de diálisis del Cantón Portoviejo. Revista Caribeña de Ciencias Sociales, 1-17.

Merino, Y. D. (2018). Estrategias de afrontamiento en pacientes con insuficiencia renal crónica hospitalizados en el área de nefrología del hospital Carlos Andrade Marín. Tesis previo a la obtención del título. Universidad Central del Ecuador, Quito. Obtenido de http://www.dspace.uce.edu.ec/bitstream/25000/14953/1/T-UCE0007-PC038-2018.pdf

Ministerio de Salud Pública MSP. (2015). Programa Nacional de Salud Renal. Obtenido de Archivo

PDF: https://aplicaciones.msp.gob.ec/salud/archivosdigitales/sigobito/tareas_seguimie nto/1469/Presentaci\%C3\%B3n\%20Di\%C3\%A1lisis\%20Criterios\%20de\%20Pri orizaci\%C3\%B3n\%20y\%20Planificaci\%C3\%B3n.pdf

Naciones Unidas. (s.f). Envejecimiento. Obtenido de Naciones Unidas: https://www.un.org/es/sections/issues-depth/ageing/index.html

Organización Mundial de la Salud . (26 de Noviembre de 2020). Actividad física. Obtenido de Organización Mundial de la Salud : https://www.who.int/es/newsroom/fact-sheets/detail/physical- 
activity\#: :text=\%C2\%BFQu\%C3\%A9\%20es\%20la\%20actividad\%20f\%C3\%A Dsica,dom\%C3\%A9sticas\%20y\%20las\%20actividades\%20recreativas.

Organizacion Mundial de la Salud OMS. (s.f). El Adulto Mayor. Obtenido de http://catarina.udlap.mx/u_dl_a/tales/documentos/lar/dionne_e_mf/capitulo1.pdf Sociedad Radiológica de Norteamérica. (s.f). RadiologyInfo.org. Obtenido de Insuficiencia (renal) de los riñones: https://www.radiologyinfo.org/sp/info.cfm?pg=kidneyfailure\#enfermedadgeneralidades 